\title{
Will CMOS Imagers Ever Need Ultra-High Speed? (INVITED)
}

\author{
Edoardo Charbon* \\ Swiss Federal Institute of Technology, Lausanne, Switzerland \\ *Email: edoardo.charbon@epfl.ch
}

\begin{abstract}
An increasing interest in new applications is driving significant research activity in the field of low-cost, ultra-high speed cameras. Such cameras must exhibit far smaller shutter time and jitter, while other properties, such as sensitivity, dynamic range, and linearity, may be traded off for speed. This tutorial paper attempts to determine when and where such trade-offs may be acceptable. Novel and conventional applications are presented for which technological advances in speed are justifiable. Implementation techniques for standard CMOS active pixel sensor technologies are outlined to enable such advances.
\end{abstract}

\section{Introduction}

Recent interest in 3D HDTV, integral vision, and tomographical imaging has prompted renewed activity in the field of high-speed imagers. We recognize two main research directions: ultra-high speed 2D imaging, including imaging with ultra-short time gating, and time-of-flight (TOF) based 3D or depth map imaging. The number of applications requiring this type of sensors is endless: from geological/industrial surveying to virtual human-computer interfaces; from material analysis to in-vivo bio-imaging; from robotic vision systems to security and biometric analysis. Systems for these applications share a set of basic specifications all referred to both time and distance measurement: Resolution $R$, accuracy $\square$, range $\left\{D_{\min }\right.$, $\left.D_{\max }\right\}$, frame rate $F R$, sensitivity and dynamic range, and shutter jitter.

Resolution $\boldsymbol{R}$ quantifies the smallest distance $d$ or time $t$ at which two objects can be separately observed. For example, if $\mathrm{R}=1 \mathrm{~mm}$, then two distinct objects separated by $1 \mathrm{~cm}$ will be resolved, while if the separation is reduced to $100 \square \mathrm{m}$, the objects will appear as one.

Accuracy $[$ relates to the standard deviation of the distance or time error that can be achieved by the device.

Range $\left\{\boldsymbol{D}_{\min }, \boldsymbol{D}_{\max }\right\}$ refers to the depth of field or the minimum and maximum distance or time of operation of the device.

Frame rate $\boldsymbol{F} \boldsymbol{R}$ is a measure of the number of complete depth maps available to the user per time unit. For example a $F R$ of 100 fps means that 100 dpeth maps per second can be achieved.

Sensitivity, generally expressed in Lux or $\mathrm{W} / \mathrm{cm}^{2}$, is also known as Noise Equivalent Power (NEP). It is defined as the minimum detectable intensity of light or number of photons $\square$ (at a given wavelength) that the pixel can detect. Typical values for a conventional CMOS Active Pixel Sensor (APS) are $0.1 \sim 10 \mathrm{Lux}$ even though a sensitivity $10^{-6} \mathrm{Lux}$ was recently reported [1]. Saturation is defined as the maximum light intensity that can be detected. Since the detection response is a continuous, linear curve, saturation is usually defined as the $5 \%$ compression point of such curve. The dynamic range of light intensity, expressed in $\mathrm{dB}$, is defined as the ratio between saturation and sensitivity. Typical values of dynamic range in CMOS APS are 30 60dB.

The dynamic range of a distance measurement is defined as the ratio between the longest measurable distance $\left(D_{\max }\right)$ and the maximum of resolution and accuracy, or $D R=$ $20 \log \left[D_{\max } / \max (R, \square)\right]$. Typical values of DR are $20 \sim 80 \mathrm{~dB}$ depending on measurement principle, quality of optical components and overall implementation setup.

An electronic shutter opens and closes upon a trigger command. The response time to the trigger, i.e. the time lag until the sensor is fully sensitive to light, is a random variable with given mean and standard deviation. The latter is defined as shutter jitter. The integration time, i.e. the time during which the sensor is fully sensitive, may be an independent random variable as well, thus also contributing to the shutter jitter. This parameter is important since it is the main limiting factor in the accuracy of depth maps when TOF methods are used.

These parameters have different impact on the imaging system, depending on whether the focus is ultra-high speed imaging, including imaging with time gating, or TOF based imaging. For ultra-high speed cameras the dominant specification is the frame rate. A reasonable dynamic range is required to allow sufficient contrast with low illumination. When time gating is used, shutter jitter is of fundamental importance since it is used to filter unwanted photons. In TOF based imagers, resolution and accuracy are important as they mainly determine the application of a particular 3D imaging system.

This tutorial paper is organized as following. In Section 2 applications requiring ultra-high speed imagers are illustrated. Section 3 presents several high-speed sensing principles known today, while the above specifications are used to evaluate different imaging systems.

\section{Applications}

\section{High-speed video imaging}

Recently, CMOS cameras have been proposed that operate at over 10kfps [2]. The speed is achieved thanks to a simultaneous pixel-level A/D conversion. However, due to the limitations of conventional optical detector design, relatively contained sensitivity and dynamic range can be guaranteed at the highest speed.

For most biological applications though high sensitivity and dynamic range cannot be traded off with speed. An example is the imaging of the diffusion of Calcium through the membrane of a neuron when exposed to a certain radiation level [3]. In this experiment resolutions of a few photons over background must be captured in integration times of a few tens of milliseconds. In these cases, cooled CCD cameras in combination with image intensifiers are generally used. However, such systems are often very expensive and difficult to calibrate. More research is currently needed to develop low- 
cost, solid-state high-speed cameras for these and other types of applications.

\section{Free Space Optical (FSO) Communications}

An emerging field of research in telecommunications is aimed at the creation of reliable optical links in the air. This approach is of course not new. In the past, the main challenge has been the mitigation of background light and the compensation of turbulences in the air that cause distortions in the signal. Recently, these issues have been addressed using detector arrays instead of a simple detector, implemented in low-cost standard CMOS. A 500kbps link based on this idea was demonstrated over a $1 \mathrm{~km}$ using a collimated $10 \mathrm{~mW}$ laser transmitter [4].

There are several apparent advantages in using optical links. First, with a simple millimetric lens one can obtain 1 mrad divergence, thus achieving the equivalent of an antenna gain of $60 \mathrm{~dB}$. In a RF transmitter such gain could be reached only with very large antennas. Such focused transmission potentially enables to fit large numbers of channels in a small volume using Space Division Multiple Access (SDMA). Second, thanks to SDMA the channel interference levels are necessarily low, thus enabling use of reduced transmission powers.

There are also severe disadvantages, the worst being the requirement of Line-of-Sight (LOS) for successful communication. However, for many applications LOS is available and the power/distance constraints are such that FSO may be the price to pay for a viable solution. The optical receiver proposed in [4], for example, is based on an array of 256 detectors. Each detector requires a NEP of $16 \square \mathrm{W} / \mathrm{cm}^{2}$ and a dynamic range of $61 \mathrm{~dB}$. To achieve the wanted bit rate the detectors must react and settle in $2 \square \mathrm{s}$. Such stringent specifications forced the designers to extend the pixel size to $335 \times 335 \square \mathrm{m}^{2}$.

\section{Geo surveying}

Surveying has traditionally involved measuring distances and/or elevations at a single point. With the introduction of laser based TOF and GPS, geo surveying tools have evolved to enable simultaneous distance estimation over a large number of points. In these applications, resolutions of millimeters over ranges of up to $100 \mathrm{~m}$ are sought. In general a single TOF based device is used in combination with mechanical scanning to cover the entire scene. An example of the depth map resulting from such a device is shown in Figure 1.
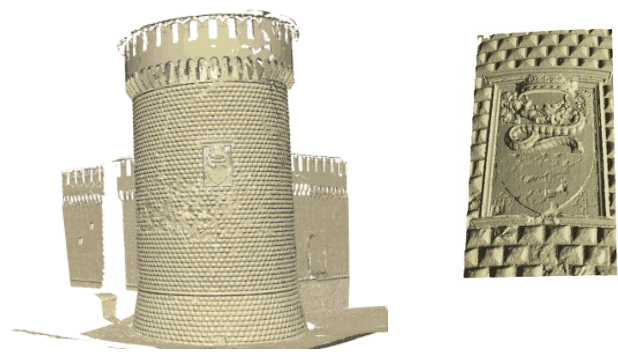

Figure 1. Survey of Castello Sforzesco in Italy, with detail.

A considerable reduction in cost could be achieved if the TOF device could be replaced with a composite array of TOF devices, so as to enable the elimination of any mechanical scanning. In this case, the system should be capable of ensuring the same range and resolution at every pixel in the array. One of the main challenges of such a system is to maintain high precision independently of the reflectivity variations within the scene. Thus, a very high distance DR may be required.

\section{Face recognition}

The literature on software tools to perform face recognition based on 2D camera images is extensive. Recently, 3D cameras have opened the way to more advanced face recognition systems [5]. The principle common to most of such approaches is to transform the surface of a face onto a representation that is invariant to lighting, orientation, and other environmental factors. An example of such canonical representation is shown in Figure 2.
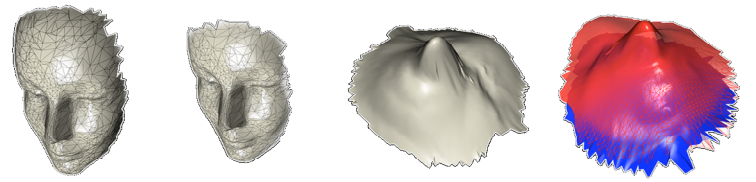

Figure 2. Process of transformation of $3 D$ faces onto invariant representations (Courtesy of R. Kimmel).

Computing geodesic distances on surfaces is a fundamental phase in the transformation process. One of the most efficient algorithms to accomplish it is known as Fast Marching on Triangulated Domains (FMTD) [6]. Figure 3 shows the invariance of a canonical representation obtained through FMTD from a set of 3D pictures of the same person.
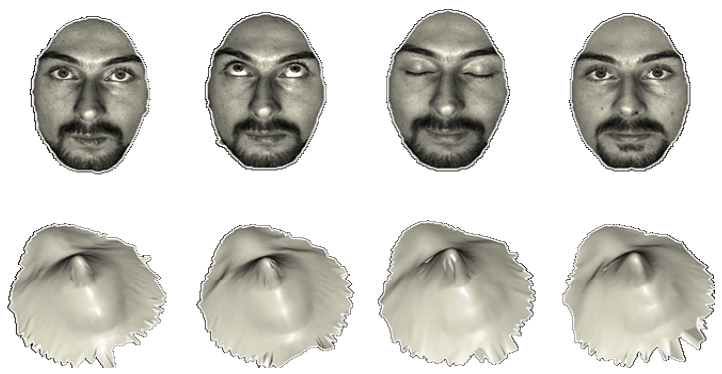

Figure 3. Canonical representations of human faces in different expressions (Courtesy of R. Kimmel).

In order for FMTD to perform at best, it is necessary that the 3D image be accurate within the entire range of operation. Acceptable values of accuracy and resolution should be approximately a millimeter, while FR should be sufficiently high to enable one to track a subject reasonably well for a few seconds, depending on the application.

\section{In-vivo brain analysis}

One of the most promising techniques to analyze the dynamics of intact human brains is based on a particular version of optical imaging. Intrinsic brain cell activity can be detected by observing changes in the optical properties of the tissue itself, which in turn affect light scattering, light absorption, and fluorescence. Optical techniques to record neural activity have been known for decades [7]. The techniques range from the evaluation of local absorption/reflectivity changes to fluorescence analysis of intrinsic chromophores at a microscopic level. However, measured variations are generally 
very small and noisy.

One of the methods that have recently emerged as a valid alternative to microscopic analysis is based on potentialsensitive dye [7]. Dye molecules bind to the external surface of excitable membranes and act as transducers, transforming changes in potential into optical intensity changes. In contrast to slow microscopic techniques, in dye based imaging intensity changes in microseconds, generally by a few photons. Figure 4 shows an example of imaging setup.

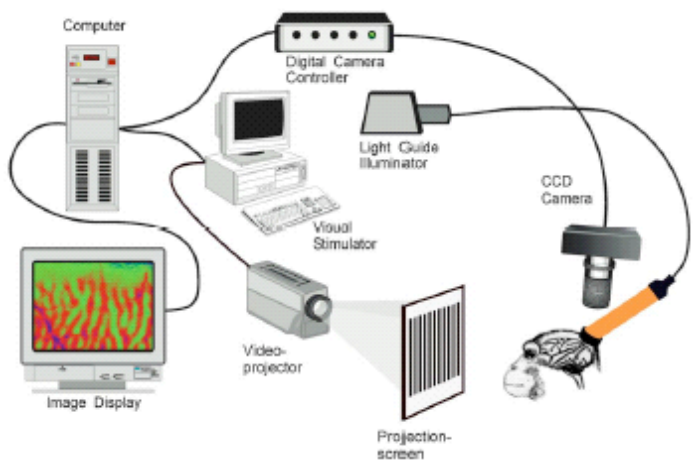

Figure 4. Optical imaging of functional maps (Courtesy of A. Grinvald).

The animal cortex is illuminated with light at $605 \mathrm{~nm}$ wavelength. The animal is shown a sequence of images and the corresponding cortical activity is captured using a conventional CCD camera in real time. The SNR is improved by means of averaging techniques.

In this case, a candidate camera for this type of imaging tasks must have a FR higher than 250fps, possibly well in the kilofps domain. Another important requirement is that its sensitivity be of the order of 10 100 photons and its dynamic range higher than $80 \mathrm{~dB}$ with a lateral resolution around 1 3Mpixel.

\section{Transillumination}

This technique has been proposed since the 1920 s as an alternative to imaging methods based on ionizing radiation, such as $\mathrm{X}$ rays. Figure 5 shows a light pulse passing through biological tissue being partially reflected (a), scattered (b), and transmitted (c).

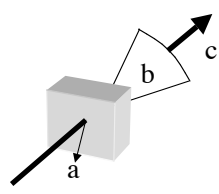

Figure 5. Transillumination principle.

The intensity of light is modulated in time depending on the thickness and nature of the tissue, however it has a consistent envelope, similar to that in Figure 6. Region (a) corresponds to the so-called ballistic photons, i.e. those photons that never experience collisions with matter. Region (b) corresponds to photons that have had one collision at most, while region (c) corresponds to scattered photons. It is generally hard to predict the path of a photon when it undergoes more than one collision [8]. Ballistic and near-ballistic photons, on the contrary, are easy to spot, provided an effective time discrimination exists.

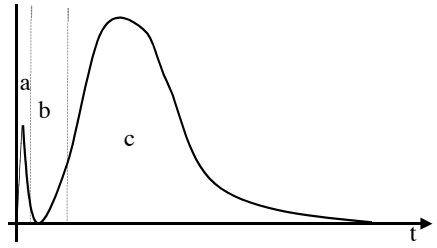

Figure 6. Envelope of the transmitted energy through biological tissue.

A typical setup to achieve such discrimination capability is based on the TOF principle using fast, low jitter optical detectors. By proper synchronization between light source and detector it is possible to discriminate ballistic and near-ballistic photons from scattered ones. Using segmentation techniques, it is then possible to eliminate all those photons that reach the detector outside the corresponding time window.

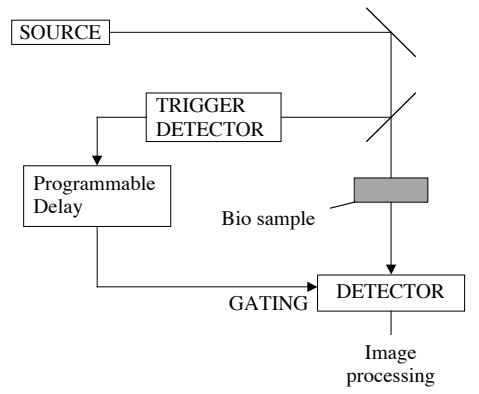

Figure 7. TOF based transillumination system.

Figure 7 shows a setup of TOF based transillumination system. Using different power levels it is possible, with this method, to identify a specific tissue based on its response to illumination [8]. Using appropriate wavelength conversion materials, it is possible to adapt optical transillumination to other types of radiation, such as X rays. Though the non-ionizing character of the approach is lost, the properties of ultra-high speed and sensitivity of the detectors may be exploited to improve tomographical resolution and to reduce the radiation doses.

\section{Fluorescence correlation spectroscopy}

Fluorescence correlation spectroscopy (FCS) is a technique used to measure transitional diffusion coefficients of macromolecules, to count fluorescent transient molecules, or to determine the molecular composition of a fluid being forced through a bottleneck or a gap. In FCS a microscopic volume, typically in the femtoliter range, is bombarded by a highly focused laser beam, thus causing the molecules in it to emit light in a well-defined spectrum. Figure 8 shows an example of optical molecular response depending on the size and diffusion pattern of the molecule.
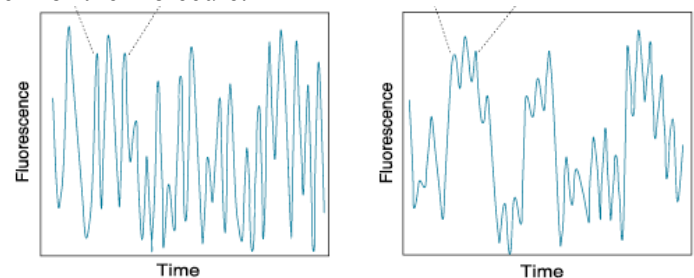

Figure 8. Optical response of molecules bombarded by highly focused laser beam. 
On the left a rapidly diffusing small molecule is depicted, on the right a slow, large molecule, with its large well-defined bursts of optical energy. The physical causes of this behavior are to be sought in the mobility of the ligands. In the first case, free fluorescent ligands are continuously entering and leaving the detection volume. In the second case, macromolecule ligands are less mobile, thus producing slower but highly correlated intensity fluctuations. Figure 9 shows an example of typical autocorrelation functions simulated for different molecules [9].

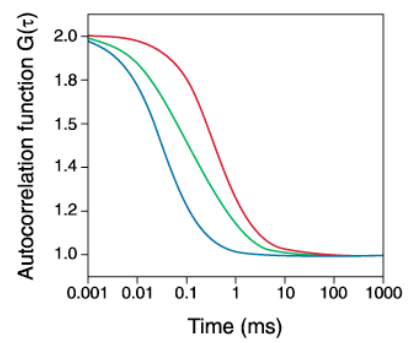

Figure 9. Autocorrelation of fluorescence response.

A tighter correlation is observable in the case of low molecular weight ligand. A macromolecule ligand generates a much more relaxed correlation. A mix of free and bound ligand is shown in the middle curve.

Recently two-photon excitation has been introduced in FCS [10]. The advantages are multifold. First, fundamental spatial confinement for the excitation can be better achieved, thus allowing one to isolate a single molecule or cluster of molecules. Second, due to the reduced optical powers in play, effects such as photobleaching and photoxicity, can be mitigated, thereby enhancing the suitability of the approach. Third, a better penetration in turbid media can be achieved, due to reduced scattering. Dual-color cross-correlation FCS measures the cross-correlation of the fluorescence intensities of two distinct dyes [11]. Thus, it becomes possible to detect different molecules without reference to their diffusion characteristics. More recently, a combination of the two methods has been reported to reduce the need for a distinct, often rather large mass ratio between the two molecule types [12].

A typical FCS setup is shown in Figure 10. A highly focused laser beam is directed towards the detection volume.

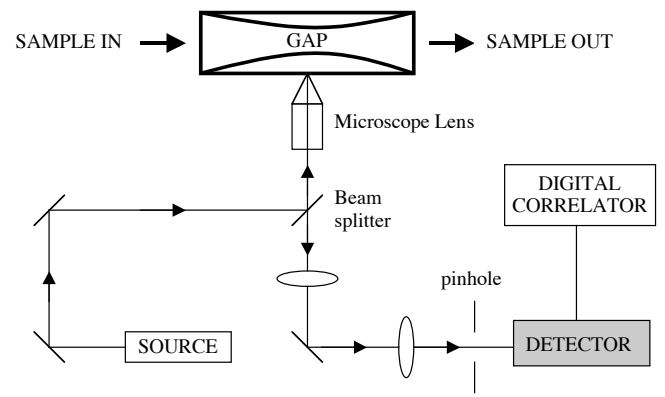

Figure 10. Basic FCS setup (gap volume not to scale).

The reflected light is applied to the high sensitivity optical sensor through a prism. A digital correlator matches the autocorrelation function with a database of known responses.
In FCS generally time resolutions of a few tens of ps and sensitivities equivalent to a few hundred photons are needed.

\section{Summary of Specifications}

Based on the above specifications, Table 1 lists the range of requirements based on the application.

\begin{tabular}{|l|c|r|r|r|r|}
\hline Application & R/ & FR & $\mathbf{D}_{\min }, \mathbf{D}_{\max }$ & \multicolumn{1}{c|}{ NEP } & DR \\
\hline HS Video & - & $10^{4}$ & - & $1000 \square$ & $20 \mathrm{~dB}$ \\
\hline $\begin{array}{l}\text { Free Space } \\
\text { Optical Com }\end{array}$ & $\square \mathrm{s}$ & - & $0.1 \sim 5 \mathrm{~km}$ & $16 \square \mathrm{W} / \mathrm{cm}^{2}$ & $>61 \mathrm{~dB}$ \\
\hline $\begin{array}{l}\text { Geo } \\
\text { Surveying }\end{array}$ & $\mathrm{mm}$ & 1 & $0 \sim 100 \mathrm{~m}$ & - & $>100 \mathrm{~dB}$ \\
\hline $\begin{array}{l}\text { Face } \\
\text { Recognition }\end{array}$ & $\mathrm{mm}$ & 30 & $0.2 \sim 2 \mathrm{~m}$ & $10000 \square$ & $50 \mathrm{~dB}$ \\
\hline IVBA & $\square \mathrm{s}$ & 250 & $20 \sim 30 \mathrm{~cm}$ & $10 \sim 100 \square$ & $80 \mathrm{~dB}$ \\
\hline $\begin{array}{l}\text { Trans- } \\
\text { illumination }\end{array}$ & $\mathrm{ps}$ & 1 & $2 \sim 3 \mathrm{~cm}$ & - & $80 \mathrm{~dB}$ \\
\hline FCS & $\mathrm{ps}$ & 1 & - & $10 \sim 500 \square$ & $40 \mathrm{~dB}$ \\
\hline
\end{tabular}

Table 1. Application based specifications.

\section{Ultra-High Speed Optical Imager Designs}

Over the last forty years, the most successful technologies used in building mainstream optical sensors have been CMOS and CCD. Many other technologies did not get as much attention mostly due to elevated production costs and limited performance. See [13] for a review. Due to lack of space, in this section we focus on CMOS APS technologies for high speed.

Since the demonstration of the first high-performance CMOS APS, both pixel pitch (hence lateral resolution), and general-purpose functions have been improved dramatically, leading to today's multi-megapixel sensors with highly controlled fixed pattern noise, Gamma correction, logarithmic intensity capture, integrated color filters, microlenses, etc. See [14] for a review.

Development of conventional CMOS APS designs for applications requiring ultra-high shutter speed and low jitter has lagged. This fact could be explained by the inherent structure of conventional pixels. In a CMOS APS, the photosensitive element of a pixel is usually implemented in one of two ways: as a photodiode or as a photogate [13]. Figure 11 shows a pixel based on a photodiode.

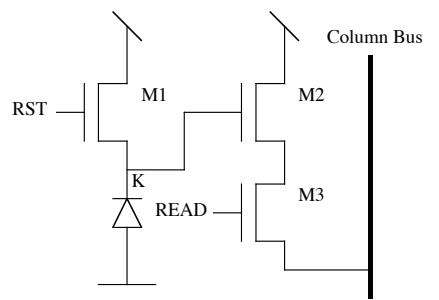

Figure 11. Schematic of a $3 \mathrm{~T}$ basic pixel topology.

In this topology, a reverse-bias diode generates a current proportional to the photon flux absorbed in and around the depletion region of the $n-p$ junction. The charges produced by the photoelectric process accumulate on the parasitic capacitance at node $K$. The resulting voltage is transferred outside the pixel via source follower M2 and switch M3. Note 
that the sole purpose of $\mathrm{M} 3$ is to select a given row whose pixel potential is to be transferred to the read-out circuitry. M1 is used to reset the photodiode at the beginning of each integration period, i.e. the period during which the pixel is active. To mitigate the errors introduced by turning M1 on and off, techniques, such as correlated and uncorrelated double sampling, have been introduced. A discussion of these techniques, in general irrelevant for speed, is omitted.

The currents generated by the photodiode when exposed to normal light intensities are in the femto- and picoampere range. Therefore, to detect the arrival time of a pulse of light with high precision, even when intensity varies abruptly, is hard. In fact, a relatively slow response can usually be observed, thus implying relatively high jitter. This fundamental limitation has generally been tackled at the pixel level.

Well-known techniques include the use of precise shutter gating and near-0 $\mathrm{dB}$ amplification on the pixel [15]. In this pixel design, shown in Figure 12, the arrival time of a light pulse is proportional to the charge accumulated in $\mathrm{C}_{\mathrm{S}}$ during the time interval $\square \mathrm{t}$ determined by the reset (RST) and shutter (SHUTTER) signals. However, nanoampere currents are still needed to obtain sub-nanosecond shutter time jitters, therefore large peak illumination power is usually specified. In addition, the distribution of highly critical timing signals throughout the sensor is critical, often limiting possible array size.

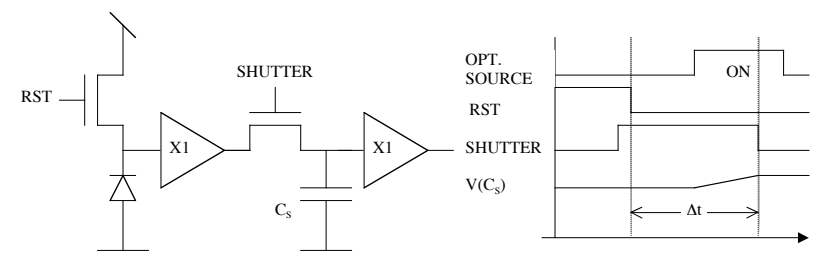

Figure 12. Low amplification, high speed detector with gating.

A viable alternative is to repeat the light source pulsing millions of times and to average the measurement of time of arrival by modulating the sensitivity of the detector in synch with the light pulses. The number of charges accumulated by the pixel, and thereby its potential, are proportional to the time the light pulses overlap with the high sensitivity window. The latter, in turn, is proportional to the arrival time to be measured [16],[17]. A possible implementation of this principle is shown in Figure 13.

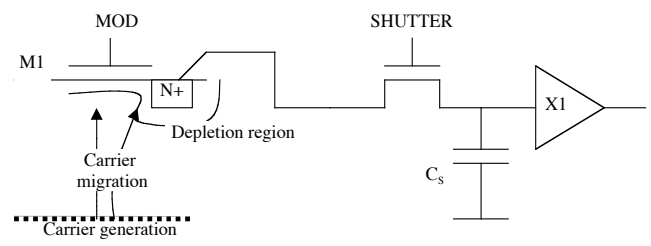

Figure 13. Modulating the sensitivity of the detector.

The photo-generated carriers are collected in the depletion region under the gate of M1. The efficiency of the collection process is modulated by forming or destroying the channel via signal MOD. After an accumulation period, during which MOD may have been varied several millions of times, the SHUTTER is turned on to transfer the accumulated voltage to the read-out circuitry. Again, the arrival time is proportional to the time overlap between the MOD signal and the carriers being generated in the substrate. This time overlap can also be seen as a phase difference between the two signals and it is proportional to the charges present in $\mathrm{N}+$ at the end of the accumulation period. Usually, a differential version of the circuit of Figure 13 is implemented to cancel contributions from the background and from slowly drifting carriers. In other implementations MOD is used as a reset and SHUTTER as a charge transfer in synch with the light source modulations. A problem with this measurement principle is the inherent ambiguity of the phase information to represent arrival time or TOF. This problem is usually addressed by performing sequences of measurements at different frequencies in combination with a successive approximation scheme. Appropriate MOD/SHUTTER signal distribution schemes need be designed and potential skews need be cancelled.

Other fast photodiode circuits, mostly intended for telecommunication applications, have been designed in the past. The literature is extensive on the subject. See [18] for a review. It is generally difficult to adapt these circuit topologies to pixel array applications. Nonetheless, there have been several successful attempts in this direction e.g. [4],[1]. In [4] for example, the classical topology of Figure 14 was modified to minimize input-referred noise and the impact of substrate noise.

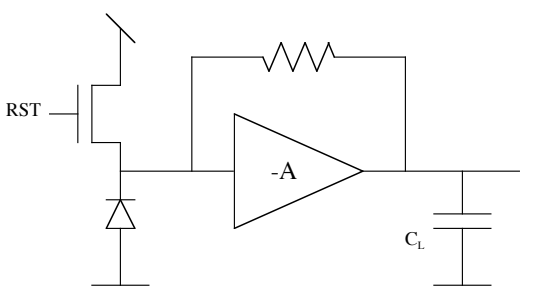

Figure 14. Classical low input impedance amplifier for ultra-small current boost.

The modifications include the use of a two-stage architecture (low noise amplifier + programmable gain amplifier), a continuous gain control feedback to avoid saturation and to boost sensitivity, a chopper-based offset reduction, and a dummy capacitance to match the photodiode parasitics for a fully differential pixel implementation. The main disadvantage of this solution is the relative size of the pixel, typically 300 to $500 \square \mathrm{m}$ even in deep sub-micron technologies.

Recently, a new generation of ultra-high speed optical devices with low timing jitter has been demonstrated in standard CMOS technology [19]. The devices, photodiodes that can be biased at and above the breakdown voltage, exploit the avalanche effect. The photo-multiplication gain, or the number of electrons generated per photon hit, can vary from one to several hundreds when the diode is biased at breakdown voltage. With a certain excess bias, the gain can be brought to infinity, thus requiring means to quench the avalanche (Geiger mode of operation). In standard CMOS technologies, quenching may be performed either via a resistor or bleeding transistor (passive quenching), or via a switch with feedback (active quenching) [19],[20].

When a comparator or appropriate buffer is added, the avalanche-generated current pulse may be converted onto a digital pulse. This topology is known as Single Photon 
Avalanche Diode (SPAD). Building usable arrays of SPADs requires means of performing a detector random access and read-out. Figure 15 shows a pixel based on SPAD technology, with row based access (READ) and independent quenching control (QNC).

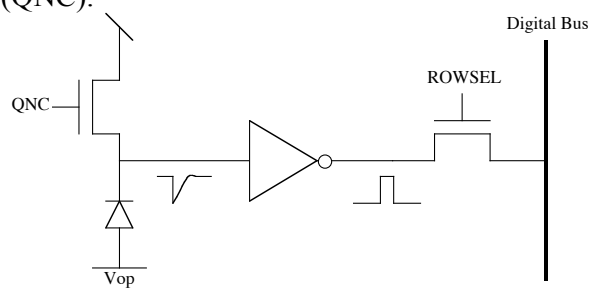

Figure 15. Pixel architecture based on 5T SPAD topology.

Due to their excellent timing jitter properties [19], SPADs (integrated in CMOS or in other technologies) have been proposed for a variety of applications, including laser distance measurement and $3 \mathrm{D}$ imaging based on TOF [21],[22],[23],[24]. However we believe that the technology has a good potential for other applications requiring highsensitivity, high-speed cameras. Nonetheless, before this type of sensors can be exploited to the fullest potential, novel pixel architectures and innovative read-out circuitries will be required, enabling capture parallelism and efficiency.

\section{Conclusions}

In this tutorial paper we have advocated research and development of ultra-high speed optical sensors. Some examples of application-specific setups requiring speed were outlined. We believe that emerging optical single photon detectors, in combination with novel read-out architectures, will revolutionize the field and, possibly, open the way to new, low-cost ultra-high speed solutions.

\section{Acknowledgments}

The author is supported by a grant of the Swiss National Science Foundation - Grant Nr.: 620-066110.

\section{References}

[1] H. Eltoukhy, K. Salama, A. El Gamal, M. Ronaghi, R. Davis, “A $0.18 \square \mathrm{m}$ CMOS $10^{-6} \mathrm{Lux}$ Bioluminescence Detection System-onChip", Proc. ISSCC, pp. 222-223, Feb. 2004.

[2] S. Kleinfelder, S. Lim; X. Liu, A. El Gamal, "A 10000 frames/s CMOS digital pixel sensor", Journal of Solid-State Circuits, pp. 2049 - 2059, Vol. 36, n. 12, Dec. 2001.

[3] Z.A. Peterlin, J. Kozloski, B-Q. Mao, A. Tsiola, R. Yuste, "Optical Probing of Neuronal Circuits with Calcium Indicators", Proc. National Academy of Sciences US, Vol. 97, n. 7, pp. 3619-3624, Mar. 2000.

[4] B. S. Leibowitz, B. E. Boser, K. S. J. Pister, "A 256-Element CMOS Imaging Receiver for Free-Space Optical Communication", Proc. Custom Integrated Circuits Conference, Oct. 2004, to appear.

[5] A. Bronstein M. Bronstein and R. Kimmel, "Expression-invariant 3d face recognition", Proc. Audio Video-based Biometric Person Authentication (AVBPA), Lecture Notes on Computer Science, pp. 62-69. Springer Verlag, 2003.

[6] R. Kimmel, J. A. Sethian, "Computing Geodesic on Manifolds", Proc. US National Academy of Science 95, pp. 8431-8435, 1998.
[7] A. Grinvald et al., "In-Vivo Optical Imaging of Cortical Architecture and Dynamics", Modern Techniques in Neuroscience Research, U. Windhorst and H. Johansson (Eds), Springer, 2001.

[8] E. B. DeHaller, "Time Resolved Breast Transillumination: Analytical, Numerical, and Experimental Study", Thesis N.1119, EPFL, 1993.

[9] K. J. Moore, S. Turconi, S. Ashman, M. Ruediger, U. Haupts, V. Emerick, A. J. Pope, "Single Molecule Detection Technologies in Miniaturized High Throughput Screening: Fluorescence Correlation Spectroscopy", Journal Biomol Screen, Vol. 4, pp. 335-354, 1999.

[10]P. Schwille, U. Haupts, S. Maiti, W. W. Webb, "Molecular Dynamics in Living Cells Observed by Fluorescence Correlation Spectroscopy with One- and Two-Photon Excitation." Biophysics Journal, Vol. 77, pp. 2251-2265, 1999.

[11]P. Schwille, F. J. Meyer-Almes, R. Rigler, "Dual-Color Fluorescence Cross-correlation Spectroscopy for Multicomponent Diffusional Analysis in Solution." Biophysics Journal, Vol. 72, pp. 1878-1886, 1997.

[12] K. G. Heinze, A. Koltermann, P. Schwille, "Simultaneous TwoPhoton Excitation of Distinct Labels for Dual-Color Fluorescence Crosscorrelation Analysis", Proc. Natl. Acad. Sci. U.S.A. 97, pp. 10377-10382, 2000.

[13]E. R. Fossum, "CMOS Image Sensors: Electronic Camera-onChip", in Trans. Electron Devices, Vol. 44, n. 10, Oct. 1997.

[14]P. M. Acosta-Serafini, I. Masaki, C. G. Sodini, “A 1/3” VGA Linear Wide Dynamic Range CMOS Image Sensor Implementing a Predictive Multiple Sampling Algorithm with Overlapping Integration Intervals", Journal of Solid-State Circuits, Vol. 39, n. 9, Sep. 2004, in print.

[15] R. Jeremias, W. Brockherde, G. Doemens, B. Hosticka, L. Listl, P. Mengel, "A CMOS Photosensor Array for 3D Imaging Using Pulsed LASER", Proc. ISSCC, pp. 252-253, February 2001.

[16] R. Lange, "3D Time-of-Flight Distance Measurement with Custom Solid-State Image Sensors in CMOS/CCD-Technology", Ph.D. Thesis, ETH-Zürich, 2000.

[17]E. Charbon and C. Bamji, "Methods for CMOS-Compatible Three-Dimensional Image Sensing Using Quantum Efficiency Modulation", U.S. Patent 6,515,740, Feb. 2003.

[18] J. Franca and Y. Tsividis (Eds.), "Design of Analog-Digital VLSI Circuits for Telecommunications and Signal Processing", PrenticeHall, Englewood Cliffs, 1993.

[19]A. Rochas, "Single Photon Avalanche Diodes in CMOS technology", Ph.D. Thesis, EPF-Lausanne, 2003.

[20] A. Rochas et al., "First Fully Integrated 2-D Array of SinglePhoton Detectors in Standard CMOS Technology", IEEE Photonics Technology Letters, Vol. 15, n. 7, pp. 963-965, Jul. 2003.

[21]B.F. Aull et al., "Geiger-Mode Avalanche Photodiodes for Three Dimensional Imaging", Lincoln Laboratory Journal, Vol. 12, n. 2 , 2002, pp. 335-350.

[22] J. Massa, G. Buller, A. Walker, G. Smith, S. Cova, M. Umasuthan, A.M. Wallace, "Optical Design and Evaluation of a ThreeDimensional Imaging and Ranging System based on TimeCorrelated Single-Photon", Applied Optics, Vol. 41, n. 6, pp. 10631070, Feb. 2002.

[23] C. Niclass, A. Rochas, P.A. Besse, and E. Charbon, "A CMOS Single Photon Avalanche Diode Array for 3D Imaging", Proc. ISSCC, pp. 120-121, Feb. 2004.

[24]C. Niclass, A. Rochas, P.A. Besse, and E. Charbon, "A CMOS 3D Camera with Millimetric Depth Resolution”, Proc. CICC, Oct. 2004 , to appear. 